Reprod. Nutr. Dévelop., 1987, 27 (4), 841-848.

\title{
Evolution postnatale de la teneur en zinc de l'épididyme et du testicule chez le rat dans les conditions normales et expérimentales
}

\author{
J. L. DELONGEAS, Marie-France HUTIN (*) (1), D. BURNEL (*), G. GRIGNON
}

Laboratoire d'Histologie, Embryologie et Microscopie électronique, Faculté de Médecine, Nancy, France.

(*) Laboratoire de Chimie Générale Appliquée à la Médecine, Faculté de Médecine, Nancy, France.

Summary. Postnatal epididymal and testicular zinc concentration in the rat under normal and experimental conditions.

Postnatal testicular and epididymal zinc concentration in the rat was investigated by means of differential pulse polarography. The zinc concentration increased gradually from birth to day 90 in the testis and up to day 60-90 in the epididymis with an abrupt increase on day 21 . No marked variation in the zinc content was observed all along the epididymal duct.

Experimental castration and efferent duct ligation were carried out in order to assess the influence of blood-borne and luminal androgens on epididymal zinc content. In prepubertal rats, unilateral castration and efferent duct ligation did not affect the zinc content of the epididymis. Moreover, zinc concentration was not affected by bilateral castration which induced very low plasma testosterone levels. These results suggested that epididymal zinc content did not depend upon endocrine testicular secretions, especially androgens. On the other hand, in adult rats efferent duct ligation and cryptorchidism resulted in about $\mathbf{5 0}$ and $70 \%$ reduction, respectively of the testicular and epididymal zinc content. A correlation was found between the absence of testicular fluid and spermatozoa or the alteration of germ cells and the decrease in epididymal and testicular zinc content.

\section{Introduction.}

Le rôle du zinc dans la fertilité des mammifères est reconnu depuis de nombreuses années. Des taux élevés de zinc sont ainsi observés dans le testicule et l'épididyme adulte, ainsi qu'au niveau des spermatozoïdes (Wetterdal, 1958 ; Gunn et Gould, 1970 ; Lindholmer et Eliasson, 1972). De plus, un déficit en zinc entraîne un retard de croissance et de maturation de l'appareil reproducteur chez

(1) Correspondance : M. F. Hutin, Laboratoire Chimie Générale Appliquée à la Médecine, Faculté de Médesine. 54505 Vandouvre-les-Nancy, France.

Reproduction, Nutrition, Développement $n^{\circ} 4$, vol. 27 (1987). -8 
le rat (Millar et al., 1958, 1960 ; Prasad et al., 1967). Toutefois il existe peu d'informations concernant l'évolution de la teneur en zinc dans le testicule et l'épididyme de rat au cours de la période postnatale.

L'objet de ce travail a été (1) de déterminer la concentration en zinc de l'épididyme et du testicule de rat, de la naissance à l'âge adulte, (2) d'examiner la répartition du zinc le long du canal épididymaire, (3) d'apprécier l'influence des androgènes (plasmatiques ou luminaux) sur la teneur en zinc de l'épididyme, en créant des modèles expérimentaux (castration, ligature des cônes efférents et cryptorchidie) car l'épididyme est un organe androgéno-dépendant (Orgebin-Crist, Danzo et Davies, 1975).

\section{Matériel et méthodes.}

Animaux et groupes expérimentaux. - 478 rats mâles, de souche Wistar (alimentation : granulés AO 4, distribués par UAR, 7, rue Galliéni, Villemoisson, 91360 Epinay-s.-Orge, concentration en zinc : $85 \mathrm{mg} / \mathrm{kg}$ ), ont été utilisés au cours de ce travail expérimental. Chez 270 rats témoins, les testicules et les segments initial, moyen et terminal de l'épididyme (Glover et Nicander, 1971) ont été prélevés au cours de la période postnatale : naissance, $10,16,21,30,40,50,60,90$ et 205 jours. 130 rats males adultes $(230-250 \mathrm{~g})$ ont subi l'une des 6 opérations suivantes : castration uni- $\left(N_{1}=25\right)$ ou bilatérale $\left(N_{2}=25\right)$, ligature uni- $\left(N_{3}=25\right)$ ou bilatérale $\left(N_{4}=20\right)$ des cônes efférents, cryptorchidie bilatérale $\left(N_{5}=20\right)$ et opération simulée $\left(N_{6}=15\right)$. 78 rats mâles âgés de 25 jours ont été castrés uni- $\left(N_{7}=28\right)$ et bilatéralement $\left(N_{8}=30\right)$ et ont eu les cônes efférents ligaturés $\left(N_{9}=20\right)$. 21 jours (groupes 1 à 6$)$ et 15 jours (groupes 7 à 9$)$ après l'opération, les testicules et les épididymes ont été prélevés, pesés et stockés à $-20^{\circ} \mathrm{C}$ jusqu'au dosage du zinc. Dans tous les cas (témoins et expérimentaux), le taux de zinc a été déterminé sur environ $200 \mathrm{mg}$ d'organe provenant d'un groupe d'animaux du même âge et traités de la même façon.

Dosage du zinc. - Le zinc a été dosé par la technique polarographique impulsionnelle différentielle que nous avons utilisée et décrite précédemment (Hutin et Burnel, 1984).

L'ion $\mathrm{Zn}^{++}$est réduit sur une électrode à goutte tombante de mercure aux alentours de $-1,05 \mathrm{~V}$ par rapport à l'électrode au calomel saturé (ECS) et donne alors en polarographie différentielle un pic dont la hauteur est proportionnelle à sa concentration. La détection limite dans un échantillon est de $0,5 \mu \mathrm{g}$ de zinc.

Les substances organiques gênantes ont été éliminées par une attaque sulfoperchlorique vers $180^{\circ} \mathrm{C}$ de l'échantilion décongelé.

Le zinc étant présent dans l'environnement, nous avons déterminé la valeur moyenne du blanc pour 16 mesures : 0,98 $\pm 0,21 \mu \mathrm{g}$. Aussi, lors de tous les dosages, il a été retranché systématiquement $1 \mu \mathrm{g}$ des valeurs obtenues (avant l'expression du résultat final en $\mu \mathrm{g} / \mathrm{g}$ tissul pour tenir compte de cet effet de la pollution. 
Dosage de la testostérone plasmatique (groupes expérimentaux). - Après étirement rachidien, du sang a été prélevé par ponction cardiaque, centrifugé et le plasma conservé à $-20^{\circ} \mathrm{C}$ jusqu'au dosage de la testostérone par RIA.

\section{Résultats.}

Dans le testicule, la teneur en zinc augmente de la naissance au $90^{\circ}$ jour, puis ne varie plus (fig. 1).

Des taux de zinc très faibles sont trouvés dans l'épididyme jusqu'au 210 jour qui correspond à une augmentation soudaine de la teneur en zinc épididymaire (fig. 2). Après 60 jours, la concentration en zinc n'évolue plus. Aucune variation significative de la teneur en zinc n'est observée le long du canal épididymaire.

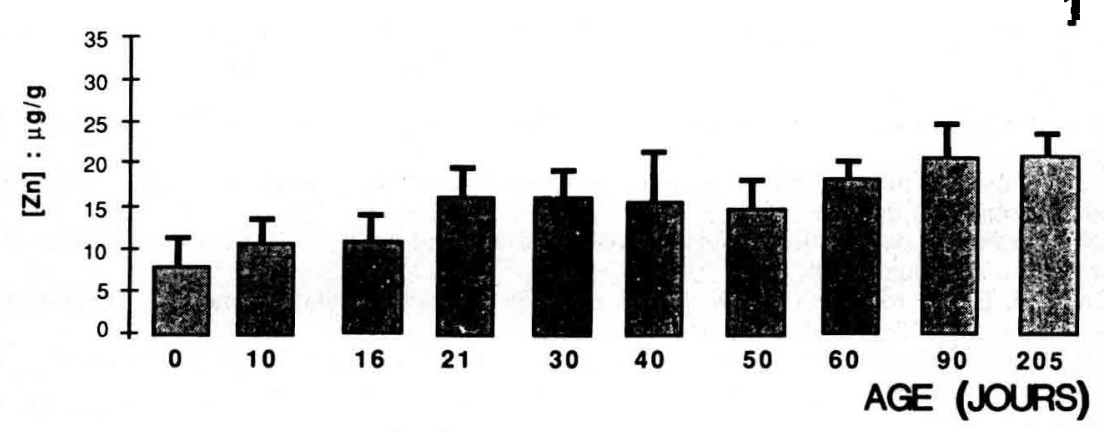

FIG. 1. - Evolution postnatale de la teneur en zinc du testicule (moyenne \pm écart type). (Nombre de mesures $=6$ pour chaque temps)

(Nombre total d'animaux $=270$ )

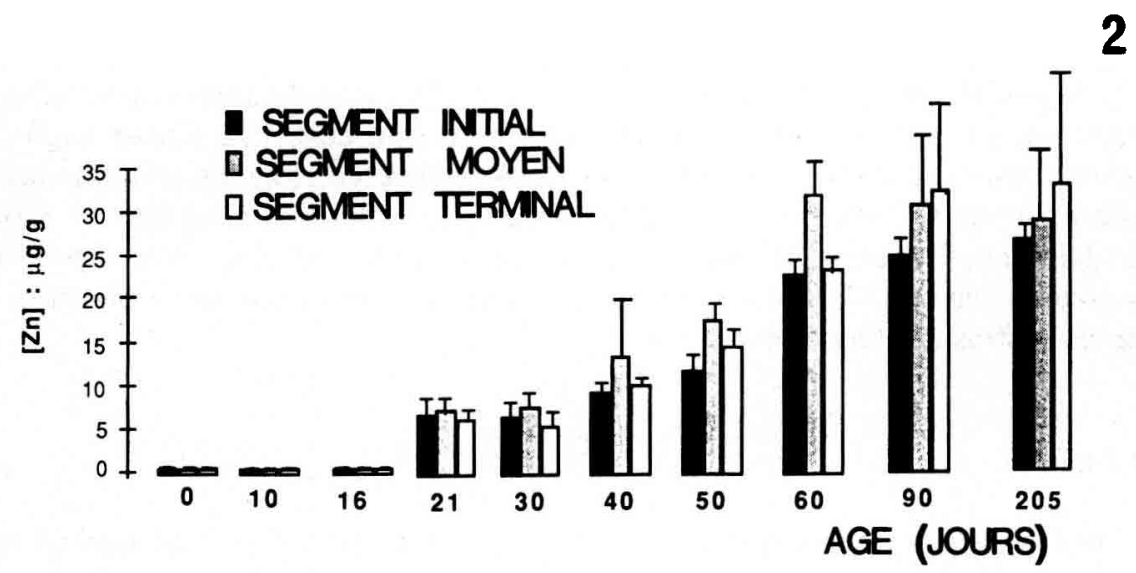

FIG. 2. - Evolution postnatale de la teneur en zinc de l'épididyme (moyenne \pm écart type). (Nombre de mesures $=6$ pour chaque temps)

(Nombre total d'animaux $=270$ ) 
En période prépubertaire, la castration unilatérale ou la ligature des cônes efférents n'entraînent aucune modification de la teneur en zinc épididymaire (fig. 3), laquelle n'est pas modifiée non plus après castration bilatérale (fig. 3), bien que les taux de testostérone circulante soient 4 fois plus faibles $(17,3 \pm 1,5 \mathrm{ng} / 100 \mathrm{ml}$ sérum ; valeur témoin $69,2 \pm 7,9 \mathrm{ng} / 100 \mathrm{ml}$ sérum$)$.

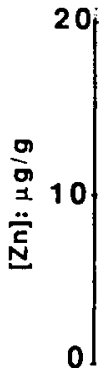

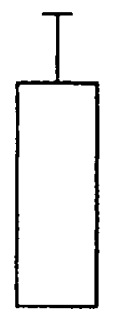

T

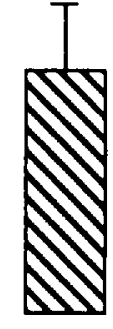

CA

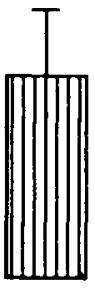

CA1/2
3

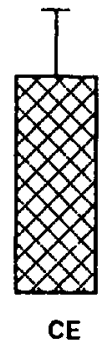

FIG. 3. - Contróle testiculaire de la teneur en zinc de l'épididyme de rat impubère (moyenne \pm écart type).

$\mathrm{T}$ : Epididyme témoin de rats de $\mathbf{4 0}$ jours (côté contralatéral témoin de rats castrés unilatéralement au $25^{\circ}$ jour) (nombre de mesures $=14$; nombre d'animaux $=28$ ).

$\mathrm{CA}$ : Epididyme de rats de 40 jours castrés bilatéralement au $25^{\mathrm{e}}$ jour (nombre de mesures $=12$; nombre d'animaux $=30$ ).

CA 1/2 : Epididyme (côté opéré) de rats de 40 jours castrés unilatéralement au $25^{\circledR}$ jour (nombre de mesures $=12$; nombre d'animaux $=28$ ).

CE : Epididyme de rats de 40 jours ayant eu les cônes efférents ligaturés au $25^{\circ}$ jour (nombre de mesures $=12 ;$ nombre d'animaux $=20$ ).

Chez le rat adulte, unilatéralement castré ou ligaturé (tabl. 1), la teneur en zinc de l'épididyme et/ou du testicule du côté opéré est significativement réduite, comparativement au côté contralatéral témoin $(p<0,001)$, bien que les taux de testostérone circulante soient comparables à ceux des témoins (respectivement $297 \pm 52 \mathrm{ng} / 100 \mathrm{ml}$ de sérum et $317 \pm 55 \mathrm{ng} / 100 \mathrm{ml}$ de sérum ; valeur témoin : $336 \pm 53 \mathrm{ng} / 100 \mathrm{ml}$ de sérum). De plus, la teneur en zinc du segment terminal de l'épididyme est significativement plus importante que celle des autres segments épididymaires $(p<0,001)$, ce qui est également observé chez les animaux ligaturés bilatéralement. Par ailleurs, la ligature des cônes efférents ainsi que la cryptorchidie bilatérales entraînent une réduction significative du zinc, d'environ $50 \%$ dans le testicule, et $70 \%$ dans l'épididyme, ce qui est également noté chez les animaux castrés bilatéralement (tabl. 1).

\section{Discussion.}

Chez le rat, la concentration en zinc augmente dans l'appareil génital mâle lors du développement postnatal. Au niveau du testicule adulte, les cellules de Sertoli renferment du zinc (Gunn et Gould, 1970) et les spermatozoïdes l'incorporent au cours des derniers stades de la spermiogenèse (Millar et al., 1961 ; Calvin 


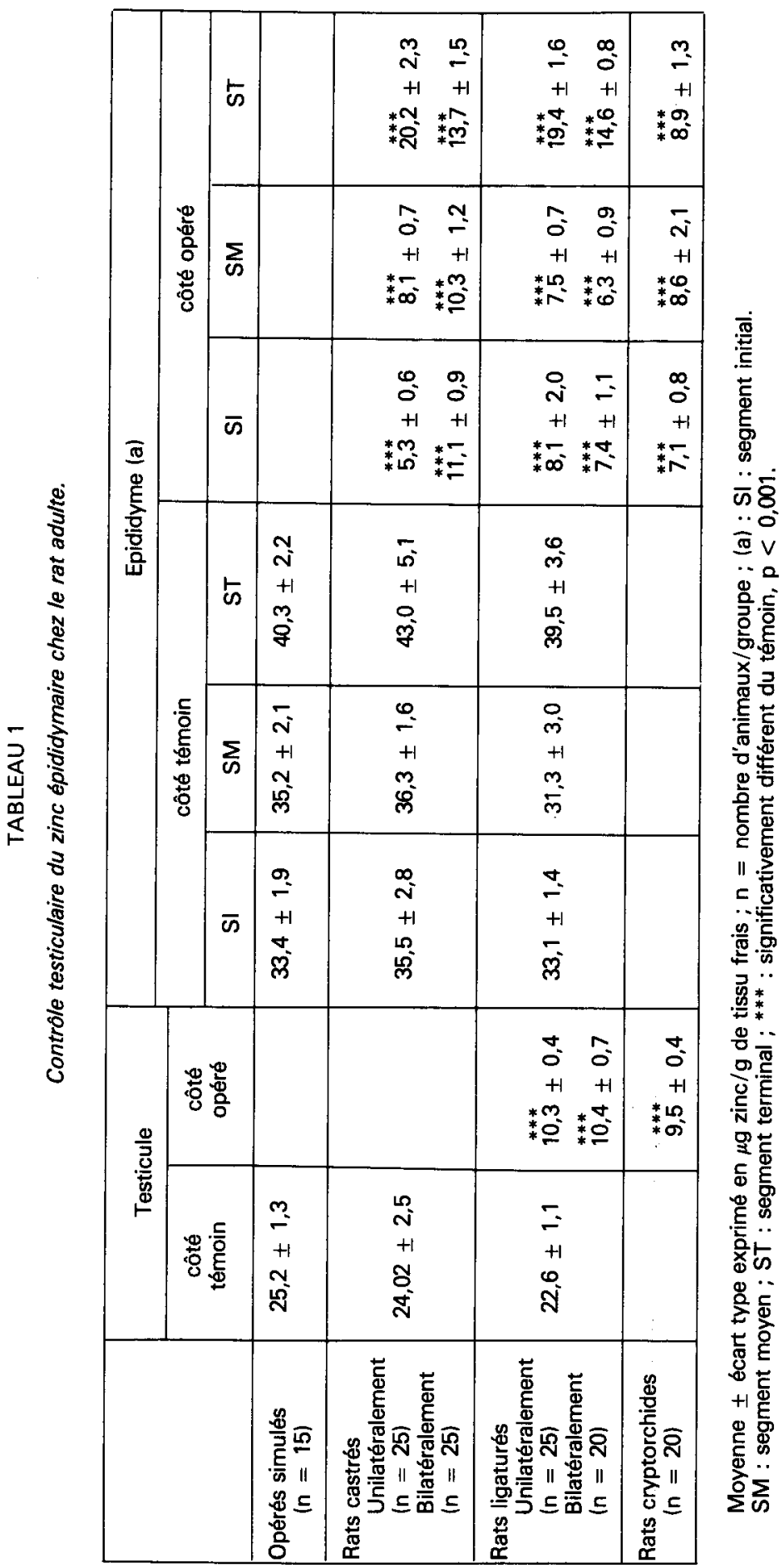


et al., 1975). L'augmentation de la teneur en zinc du testicule, de la naissance à 21 jours, puis ultérieurement, est imputable respectivement, à la multiplication des cellules de Sertoli pendant les trois premières semaines de la vie postnatale (Orth, 1982), et au déroulement de la spermatogenèse (Gondos, 1977).

Sun et Flickinger ont montré que la différenciation cellulaire de l'épididyme de rat s'effectuait entre 16 et $\mathbf{4 0}$ jours. Nous constatons une augmentation de la teneur du zinc de l'épididyme pendant cette période, suggérant une participation du zinc au développement de l'épididyme. Des travaux ont montré que les enzymes ADN et ARN polymérases impliquées dans les processus cellulaires de replication et de différenciation nécessitent la présence du zinc pour leur fonction (Williams et Loeb, 1973 ; Underwood, 1981), et qu'un déficit en zinc entraîne un retard de maturation et de croissance des organes reproducteurs du rat (Millar et al., 1960 ; Prasad et al., 1967).

L'augmentation brutale, au 21॰ jour, de la teneur en zinc de l'épididyme coïncide avec l'élévation des taux plasmatiques de testostérone (Resko, Feder et Goy, 1968 ; Dohler et Wuttke, 1975) et à l'arrivée de fluide testiculaire dans la lumière du canal épididymaire (Knorr, Vanha-Perttula et Lipsett, 1970). La réalisation expérimentale de castration et de ligature des cônes efférents permet d'apprécier l'influence des androgènes circulants et luminaux sur la teneur en zinc de l'épididyme. Les résultats obtenus chez le rat impubère montrent que la concentration en zinc dans l'épididyme n'est pas régulée par les androgènes d'origine testiculaire, car leur suppression totale par castration bilatérale $n$ 'altère pas la teneur du zinc épididymaire. De même, chez l'adulte, des taux normaux de testostérone circulante ne suffisent pas à maintenir la valeur du zinc épididymaire du côté opéré chez les rats castrés ou ligaturés unilatéralement, ce qui confirme les résultats de Snaith, Hay et Levy (1971) qui n'avaient pu ramener à des valeurs normales le taux de zinc de l'épididyme de rat adulte castré par l'administration exogène de testostérone. Par ailleurs, on ne peut exclure l'influence possible d'autres hormones (œstrogènes ou prolactine) sur le métabolisme du zinc épididymaire, d'autant que des récepteurs pour ces deux hormones ont été mis en évidence au niveau de l'épididyme (Orgebin-Crist et Djiane, 1979 ; Danzo, St-Raymond et Davies, 1981 ; Schleicher et al., 1984). La réponse variable aux traitements est à relier avec l'état d'avancement de la spermatogenèse. En effet, chez le rat adulte, le zinc incorporé dans les spermatozoïdes (Calvin, Hwang et Wohlrab, 1975) représente une fraction très importante du zinc épididymaire. Ainsi, l'absence de spermatozoïdes dans la lumière du canal épididymaire, cas du segment initial et moyen chez les rats castrés, ligaturés et cryptorchides, s'accompagne d'une chute de $70 \%$ de la valeur du zinc épididymaire; par ailleurs, la présence de quelques spermatozoïdes et spermiophages au niveau du segment terminal de l'épididyme des rats unilatéralement castrés ou ligaturés maintient le taux de zinc à une valeur plus élevée. De même, l'altération de la spermatogenèse observée chez les rats ligaturés ou cryptorchides s'accompagne parallèlement d'une chute significative du taux de zinc testiculaire.

Au cours du développement, et chez l'adulte, nous ne constatons pas de variations dans la teneur en zinc le long du canal épididymaire, à l'inverse de ce que l'on observe avec d'autres ions, sodium ou potassium, dont les variations 
dans le fluide le long du canal épididymaire sont le reflet de phénomènes d'absorption et de secrétion de l'épithélium (Brooks, 1983). Nos observations concernant la variation du zinc dans l'épididyme sont identiques à celles rapportées chez le singe Rhésus adulte (Roy et al., 1974), et sont plus en faveur d'une prédominance du zinc dans les spermatozoïdes que dans le fluide épididymaire.

En conclusion, chez le rat, le zinc se fixe progressivement au niveau du testicule et de l'épididyme. Dans l'épididyme, sa concentration ne semble pas dépendre directement des androgènes testiculaires suggérant l'intervention d'autres facteurs, hormonaux ou non. Chez l'adulte, le taux de zinc du testicule et de l'épididyme dépend étroitement du bon déroulement de la spermatogenèse et de la présence de spermatozoïdes dans la lumière du canal.

Reçu en décembre 1986. Accepté en avril 1987.

\section{Références}

BROOKS D. E., 1983. Epididymal functions and their hormonal regulation. Aust. J. biol. Sci., 36, 205-221.

CALVIN H. I., HWANG F. H. F., WOHLRAB H., 1975. Localization of zinc in a dense fiber connecting piece fraction of rat sperm tails analogous chemically to hair keratin. Biol. Reprod., 13, 228-239.

DANZO B. J., ST-RAYMOND P. A., DAVIES J., 1981. Hormonally responsive aeras of the reproductive system of the male guinca pig. III. Presence of cytoplasmic estrogen receptors. Biol. Reprod., 25, 1159-1168.

DOHLER K. D., WUTTKE W., 1975. Changes with age in levels in serum gonadotropins, prolactin and gonadal steroids in prepubertal male and female rats. Endocrinology, 97, 898-907.

GLOVER T. D., NICANDER L., 1971. Some aspects of structure and function in the mammalian epididymis. J. Reprod. Fertil, suppl. 13, 39-50.

GONDOS B., 1977. Testicular development. In JOHNSON A. D., GOMES W. R. The testis, Acad. Press, New York, IV, 1-27.

GUNN S. A., GOULD T. C., 1970. Cadmium and other mineral elements. In JOHNSON A. D., GOMES W. R., VAN DEMARK N. L. The testis, Acad. Press, New York, III, 377-481.

HUTIN M. F., BURNEL D., 1984. Dosage du zinc dans l'eau et certains milieux biologiques par polarographie impulsionnelle. Ann. Falsif. Expert. Chim., 833, 587-593.

KNORR D. W., VANHA-PERTTULA T., LIPSETT M. B., 1970. Structure and function of rat testis through pubescence. Endocrinology, 86, 1298-1304.

LINDHOLMER C., ELIASSON R., 1972. Zinc and magnesium in human spermatozoa. Int. J. Fertil., 17, 153-160.

MILLAR M. J., ELCOATE P. V., FISCHER M. I., MAWSON C. A., 1960. Effect of testosterone and gonadotrophin injections on the sex organ development of zinc deficient male rats. Can. J. Biochem. Physiol., 38, 1457-1465.

MILLAR M. J., FISCHER M. I., ELCOATE P. V., MAWSON C. A., 1958. The effects of dietary zinc deficiency of the reproduction system of male rats. Can. J. Biochem. Physiol., 36, 557-569.

MILLAR M. J., VINCENT N. R., MAWSON C. A., 1961. An autoradiographic study of the distribution of zinc 65 in rat tissues. J. Histochem. Cytochem., 9, 111-125.

ORGEBIN-CRIST M. C., DANZO B. J., DAVIES J., 1975. Endocrine control of the development and maintenance of sperm fertilizing ability in the epididymis. In Handbook of Physiology, section 7: Endocrinology, Vol. 5 : Male reproductive system, pp. 319-338. Eds R. O. GREEP \& E. B. ASTWOOD. Am. Physiol. Soc., Washington D. C. 
ORGEBIN-CRIST M. C., DJIANE J., 1979. Properties of a prolactin receptor from the rabbit epididimys. Biol. Reprod., 21, 135-140.

ORTH J. M., 1982. Proliferation of Sertoli cells in fetal and postnatal rats : a quantitative autoradiographic study. Anat. Rec., 203, 485-492.

PRASAD A. S., OBERLEAS D., WOLFE P., HORWITZ J. P., 1967. Studies on zinc deficiency : changes in trace elements and enzyme activities in tissues of zinc - deficient rats. J. clin. Invest., 45, 549-557.

RESKO J. A., FEDER H. H., GOY R. W., 1968. Androgen concentrations in plasma and developing rats. J. Endocrinol., 40, 485-491.

ROY S. K., SETTY B. S., CHANDRAY H., KAR A. B., 1974. The effect of efferent duct ligation on the uptake of $\mathrm{Zn} 65$, by the epididymis and vas deferens of rhesus monkeys (Macaca mulattal. Acta endocr., 77, 186-192.

SCHLEICHER G., DREWS U., STUMPF W. E., SAR M., 1984. Differential distribution of dihydrotestosterone and estradiol binding sites in the epididymis of the mouse. An autoradiographic study. Histochemistry, 81, 139-147.

SNAITH S. M., HAY A. J., LEVY G. A., 1971. The relationship between the mannosidase activity and the zinc content of mammalian sex organs. J. Endocrinol., 50, 659-667.

SUN E. L., FLICKINGER C. J., 1979. Development of cell types of regional differences in the postnatal rat epididymis. Am. J. Anat., 154, 27-56.

UNDERWOOD E. J ., 1981. Trace metals in human and animal health. J. human nutr., 35, 37-48.

WETTERDAL B., 1958. Experimental studies on radio-active zinc in the male reproductive organs of the rat. Acta radiol., suppl. 156, 1-79.

WILLIAMS R. O., LOEB L. A., 1973. Zinc requirement for DNA replication in stimulated human lymphocytes. J. Cell Biol., 58, 594-601. 